\title{
Effectiveness of percutaneous tibial nerve stimulation in the treatment of overactive bladder syndrome
}

REVIEW

\section{Liesbeth $L$ de Wall John PFA Heesakkers}

Department of Urology, Radboud University Medical Centre, Nijmegen, The Netherlands

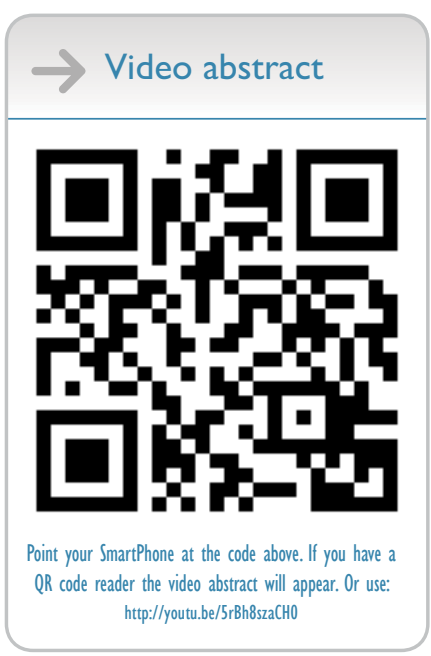

Correspondence: Liesbeth $\mathrm{L}$ de Wall; John PFA Heesakkers

Department of Urology, Radboud University Medical Centre, Geert Grooteplein Zuid I0, 6500 HB Nijmegen, The Netherlands

Tel +3I 24 36 | 67I2

Email liesbeth.dewall@radboudumc.nl; john.heesakkers@radboudumc.nl
This article was published in the following Dove Press journal:

Research and Reports in Urology

14 August 2017

Number of times this article has been viewed

\begin{abstract}
Overactive bladder syndrome (OAB) is a common condition affecting adults and children worldwide, resulting in a substantial economic and psychological burden. Percutaneous tibial nerve stimulation (PTNS) is derived from acupuncture used in Chinese traditional medicine and was first described in the early 1980s. It is a neuromodulation technique used to modulate bladder function and facilitate storage. Being a minimally invasive, easily applicable, but time-consuming treatment, future developments with implantable devices might be the solution for the logistical problems and economic burden associated with PTNS on the long term. This nonsystematic review provides a current overview on PTNS and its effectiveness in the treatment of $\mathrm{OAB}$ for both adults and children.
\end{abstract}

Keywords: overactive bladder, percutaneous tibial nerve stimulation, neuromodulation, electrical stimulation

\section{Introduction}

Overactive bladder syndrome (OAB) is a common condition defined as urgency to void, usually accompanying frequency and nocturia, with or without urge urinary incontinence (UUI), in the absence of urinary tract infection (UTI) or other obvious pathology. ${ }^{1,2} \mathrm{OAB}$ with or without UUI affects millions worldwide, with a prevalence ranging from $1.5 \%$ to $36.4 \%$ resulting in a substantial economic and psychological burden. ${ }^{3,4}$ Health-related quality of life (QoL) is usually negatively affected, and patients with OAB experience more anxiety and depression compared to healthy controls. ${ }^{4-7}$ In these patients, social stigmatization frequently leads to less self-esteem and impaired interpersonal interactions. ${ }^{8,9}$ Although the etiology of OAB is multifactorial, some genetic predisposition might exist, as there is a reported 2.8 times increased risk for children with mothers suffering from OAB. ${ }^{10}$

Neuromodulation, like sacral nerve stimulation (SNS) and percutaneous tibial nerve stimulation (PTNS) of the lower urinary tract, is a second-line treatment option for refractory $\mathrm{OAB}$. In theory, neuromodulation should be minimally invasive, easily applicable, and not cause unnecessary embarrassment by stimulating specific areas of the body, for example, the genital area. Furthermore, sustainability and cost-effectiveness are important in view of the competitiveness with other treatments.

This nonsystematic review presents a summary of the history and theories on the pathophysiology behind neuromodulation, the clinical results, and latest developments in PTNS. The aim was to provide an up-to-date overview on PTNS and its effectiveness in the treatment of OAB. 


\section{Materials and methods}

We searched the databases PubMed, MEDLINE, Cochrane, and Embase for relevant English-language articles. In addition, citations from the primary references were scrutinized for relevant articles that the databases could not locate. A combination of keywords used for the search included: percutaneous or posterior tibial nerve stimulation, neuromodulation, effectiveness, long-term outcome, patient perspectives, prognostic factors, cost-effectiveness, and implantable stimulator.

\section{Results}

\section{General principle of neuromodulation}

The innervation of the lower urinary tract comes from the lumbar, sacral, and coccygeal segmental nerves originating from L2-S4. A large network of both afferent and efferent fibers is formed after exiting the spinal cord, innervating all the pelvic organs. The sciatic nerve is composed of fibers from L4 to S3 and descends down toward the lower extremities. ${ }^{11}$ One of its distal branches is the posterior tibial nerve (PTN). Neuromodulation is postulated to be the effect of cross-signaling between sympathetic and parasympathetic postganglionic nerve terminals and synapses, causing alteration of nerve signals involved in the voiding reflex. de Groat et $\mathrm{al}^{12}$ described this neurophysiological process and the neural circuits involved in controlling the lower urinary tract. Stimulation of peripheral nerves and subsequent "cross-talk" at the level of the postganglionic neuroeffector junctions can modulate transmission. This implies that stimulating one area of the innervations system seems to alter the nerve behavior of other systems, leading to alteration in bladder function by stimulating peripheral nerves. The pudendal nerve, the dorsal genital nerve, and the PTN are examples of such peripheral nerves that can affect bladder behavior. Stimulating overlying skin or dermatomes, instead of actual nerves, is another option of peripheral neuromodulation. ${ }^{13-16}$

\section{Effects on the peripheral nervous system}

The mechanism behind neuromodulation is still not completely understood. Alteration of the afferent and efferent pathways between the brain, brain stem, and pelvic organs are thought to modulate the voiding reflex and facilitate storage. Symptoms of OAB (including UUI) may represent the clinical expression of an alteration of the pelvic neuromuscular environment via changes in the inhibitory and excitatory signals of the voiding reflex; this has been confirmed in animal studies. A normal spinal-brain stem-spinal reflex is seen in cats with an intact central nervous system (CNS), activated by non-nociceptive $\mathrm{A} \delta$ bladder afferent fibers which pass through the supraspinal relay stations in the periaqueductal gray (PAG) to the pontine micturition center (PMC). The PMC activates the efferent pelvic nerve which results in voiding. ${ }^{12,17}$

This reflex is present even in decerebrated animals, but is blocked by the transection of the spinal cord because, then, the afferent signals from the bladder cannot go up and the efferent signals from the PMC to the bladder cannot go down. If the spinal transection is distal to the sacral segments, irrigation of the bladder by diluted acetic acid (AA) unmasks reflex contractions mediated by spinal reflex circuitry activated $C$ fiber bladder afferents instead of non-nociceptive A $\delta$ bladder afferent fibers. ${ }^{18}$ It is postulated that detrusor overactivity (DO) in OAB is mediated through these C fibers. ${ }^{19,20}$

Animal studies have shown different effects of SNS, pudendal nerve stimulation (PNS) or tibial nerve stimulation (TNS) on voiding reflex pathways each including different neurotransmitter mechanism. ${ }^{21,22}$ In decerebrate cats under anesthesia, DO was evoked by direct electrical stimulation (ES) of the PMC, activating the excitatory efferent output from PMC to the bladder. Subsequently, PNS or TNS followed. PNS but not TNS was able to inhibit PMC-induced DO. Furthermore, propanolol (a non-selective B-adrenergic receptor antagonist) completely eliminated PNS-induced inhibition of DO. On the contrary, TNS facilitated PMC-induced DO and was blocked by propanolol. The authors concluded that B-adrenergic receptors are mainly involved in PNS-induced inhibition of DO acting on the efferent pathway of the voiding reflex while TNS might use the same receptors for the opposite effect, that is, facilitation of DO. Instead of influencing the efferent pathway of the voiding reflex like PNS, the inhibitory effect of DO after TNS is believed to be the effect of modulation of the afferent pathway. Strong inhibition of the ascending sensory pathway and weak excitation of the excitatory efferent pathway will result in the overall inhibition of DO seen after TNS. ${ }^{22}$

While B-adrenergic receptors are thought to play a major role in PNS, opioid receptors and endogenous enkephalins seem to be important in the mechanism behind TNS. In cats with an intact CNS, intravenous naloxone (opioid receptor antagonist) blocked the TNS-induced inhibition of DO after AA bladder installation, the latter representing a model for OAB. Nalaxone was unable to block TNS-induced inhibition of normal reflex bladder activity provoked by saline bladder instillation. ${ }^{18}$ Tai et al also studied the role of nalaxone administration in TNS-induced inhibition of DO in cats. Nalaxone was able to block TNS-induced inhibition of DO after AA installation but, furthermore, did not change cystometric 
bladder capacity (CMC). On the contrary, nalaxone was unable to alter TNS-induced normal bladder reflex activity after saline installation but reduced CMC instead. ${ }^{23}$ Nalaxone could not alter PTNS-induced inhibition but reduced CMC, indicating that the voiding reflex was inhibited by the activation of opioid receptors and endogenous enkephalins. On the contrary, nalaxone suppressed PTNS-induced inhibition after AA installations but did not alter $\mathrm{CMC}$, indicating that the enkephalinergic inhibition was inactive during AA-induced DO, but was activated by PTNS. This supports the idea that bladder activity in OAB is mediated through afferent $\mathrm{C}$ fibers being nociceptive, while normal reflex bladder activity is mediated through A $\delta$ afferent fibers. Saline installations activate non-nociceptive $A \delta$ afferent fibers which, in their turn, trigger a spinobulbospinal bladder reflex transmitted through the PAG and PMC. On the other hand, AA irritation of the bladder activates nociceptive $\mathrm{C}$ fibers that facilitate the supraspinal reflex. Enkephalinergic mechanisms are not involved in the control of the $\mathrm{C}$-fiber-mediated spinal reflex, but are involved in the inhibitory modulation of this reflex induced by PTNS. ${ }^{23}$

Li et $\mathrm{al}^{24}$ implanted a sacral nerve stimulator in 7 pigs and evoked DO bladder by AA instillations. Consecutive cystograms at baseline, after infusion of AA and after SNS, were performed with or without intravenous naloxone and tramadol infusion. Remarkably, SNS combined with tramadol had a significantly better effect on CMC than SNS alone, suggesting the anti-nociceptive effect and subsequent inhibition of DO while naloxone blocked the effect of SNS leading to a decreased $\mathrm{CMC}^{24}$

\section{Effects on the CNS}

Blok et al studied the effects of acute and chronic SNS on the brain using positron emission tomography images. ${ }^{25}$ Areas located in the right postcentral gyrus, left parietal cortex, right insula, and medial prefrontal cortex showed increased cerebral blood flow during acute SNS in newly implanted patients. Furthermore, increased activation in the ventromedial-orbitofrontal cortex and decreased activation in the left medial cerebellum occurred. This suggests modulation of areas involved in sensorimotor learning when starting SNS. During chronic SNS, there was decreased activity in the cerebellum, midbrain, and adjacent thalamus and limbic cortical areas, areas previously implicated in the control of bladder contractions, awareness of bladder filling, and initiation of voiding. This implies that different areas in the brain are involved in the learning process and start of SNS, which are taken over by other areas in the brain as time passes, resulting in a shift from dysfunctional to normal control of the voiding reflex.

Information processing in the brain after peripheral nerve stimulation can be visualized measuring somatosensory evoked potentials (SEPs). Especially long-latency somatosensory evoked potentials (LL-SEPs) seem to provide information on the function of somatosensory associative cortical structures. The presence of reproducible LL-SEPs are likely to be responsible for the neuroplastic changes induced by neuromodulation. ${ }^{26}$ Finazzi-Agro et $\mathrm{al}^{27}$ studied this more thoroughly. Both short-latency SEP (SL-SEP) and LL-SEPs were recorded after peripheral or sham stimulation. ${ }^{27}$ Peak latency and peak-to-peak amplitude of so-called P80, P100, and P200 waves were measured at baseline and at the end of (sham) stimulation. Mean latency of the previously mentioned waves and the mean amplitude of P200 waves did not change significantly. However, the amplitude of LL-SEP changes of especially P80 and P100 waves in the active PTNS group was significantly higher as compared to the sham group. The recorded P80 and P100 amplitude increase suggests long-term modifications in the synaptic efficiency of the somatosensory pathway. Long-term potentiation and depression of excitatory synaptic transmission can contribute to experience-dependent modifications of the brain, including learning and memory. ${ }^{27}$ This confirms the idea of the re-organization of the cortical network as a result of peripheral neuromodulation.

\section{History of PTNS}

Peripheral neurostimulation is derived from techniques used in traditional Chinese medicine, better known as acupuncture. Acupuncture was already practised during the Stone Age. The earliest writings about "stone needles" (called Pien in Chinese) date from about 500 BC. Puncturing specific points was believed to restore "the energetic harmony" of the body. ${ }^{28}$ In 1673, a Dutch physician (Wilhelmus ten Rhyne) discovered this Eastern traditional way of medicine which he published in a book entitled Dissertatio de Arthritide: Mantissa Schematica: De Acupunctura: Et Orationes tres. In this book, he was the first Western person to describe the technique he called "acupunctura," in which needles were used to treat diseases. One of the most commonly used acupuncture points is the San-Yin-Jiao point or Spleen 6 (SP-6). This is located on the medial side of the lower leg, about 4 finger breadths cephalad to the medial malleolus. The location of the SP-6 point and the organs affected by its stimulation have remarkable similarities with PTNS (Figure 1). When an electrical current is applied to the acupuncture needle, the technique is called electrical acupuncture. Especially when electroacupuncture 


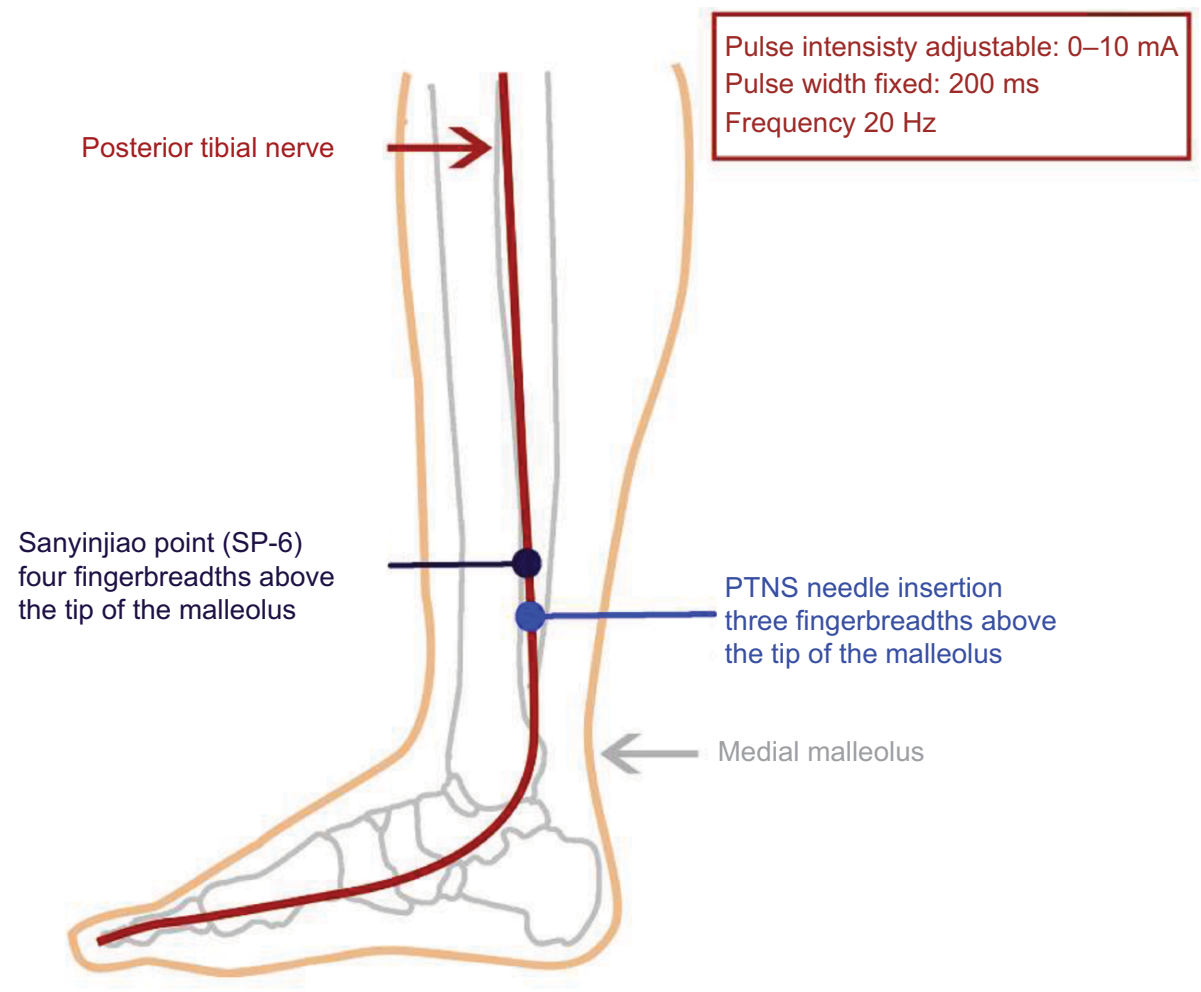

Figure I Drawing showing the location of the Sanyinjiao point, or Spleen 6 (SP-6), the percutaneous tibial nerve stimulation (PTNS) point, and the technical details.

is performed with similar stimulation parameters $(2-15 \mathrm{~Hz}$, 10-20 mA), it resembles PTNS. ${ }^{29}$ The main difference lies in the actual anatomical substrate used in PTNS, instead of "energy pathways" stimulated in acupuncture.

Before the introduction of PTNS, SNS was the main neuromodulation technique used. SNS was developed by Tanagho and Schmidt in the late 1980s, and the first device was implanted in Europe in 1989. At first, it was believed that stimulation would lead to actual contraction of the pelvic floor and sphincter complex to prevent urinary leakage. Instead, during urodynamic studies, it actually led to the inhibition of DO. Nowadays, SNS is an evidence-based clinical tool for patients with $\mathrm{OAB}$ or non-obstructive retention. ${ }^{30,31}$ After SNS was introduced, the next aim was to develop more easily accessible and less invasive techniques, such as PTNS.

McGuire et al were the first to describe PTNS in 1983. In 22 patients with neurogenic OAB, TNS was applied and $87 \%$ showed complete or partial improvement of their symptoms. ${ }^{32}$ Subsequently, Stoller et al further developed PTNS, also known as Stoller afferent nerve stimulation (SANS), as a treatment for OAB in pig-tailed monkeys and, later, in humans, with promising results. ${ }^{33}$ This new initiative was the start of the worldwide development and exploration of PTNS.

\section{Technique}

PTNS is given in supine position with the medial malleolus pointing upwards. In children, previous administration of a topical anesthetic agent (eg, lidocaine) can help to reduce pain and fear associated with needle insertion. A 34-gauge stainless steel needle is inserted $\sim 3$ finger breadths cephalad to the medial malleolus, between the posterior margin of the tibia and soleus muscle (Figure 1). The goal is to place the tip of the needle close to the PTN without actually touching it. Given the varying size of ankles, the optimal depth and angulation may be varied. On average, insertion depth is about $2-4 \mathrm{~cm}$ with an angulation of $60^{\circ}-90^{\circ}$. A stick electrode is placed on the same leg near the arch of the foot. The needle and the electrode are connected to a low voltage $(9 \mathrm{~V})$ stimulator (Urgent $\mathrm{PC}^{\circledR}$; Cogentix Medical Inc., Minnetonka, MN, USA) with an adjustable pulse intensity of $0-10 \mathrm{~mA}$, a fixed pulse width of 200 microseconds, and a frequency of $20 \mathrm{~Hz}$ (Figure 1).

Stimulation of the PTN leads to effects in both efferent and afferent nerve fibers. Flexion of the great toe, or fanning, is a result of an efferent effect. The sensory afferent effect is a radiation tickling sensation of the foot sole. During the initial test stimulation, the amplitude is slowly increased until the large toe starts to curl, or toes start to fan. If the patient complains about discomfort or "buzzing" 
immediately around the needle site, the needle may not be deep enough. In contrast, the needle may be too close to the PTN if the stimulation is extremely uncomfortable. Needle repositioning or reinsertion at the ipsilateral or contralateral ankle is advised. Once optimal position is assured, stimulation is applied at an intensity level well tolerated by the patient and can be increased or decreased during the treatment. ${ }^{34}$

Most treatment schedules consist of 12 outpatient consecutive treatment sessions lasting 30 minutes each, given $1-3$ times per week. ${ }^{35,36}$

\section{Clinical results}

Since its introduction, many clinical trials have used PTNS to treat either $\mathrm{OAB}$ or non-obstructive urinary retention. Outcomes in these studies are mainly based on frequency voiding charts (FVCs) and QoL questionnaires. ${ }^{34,37-39}$ Overall subjective success, defined as improved QoL or willingness to continue treatment, was found in $56 \%-63 \%$. Overall objective success with $\geq 50 \%$ decrease in urge or UUI and $25 \%$ reduction in daytime and/or nighttime frequency was found in $33 \%-71 \%$. $^{34,37-39}$

Urodynamics done to provide more objective data show conflicting results. PTNS performed in eight neurological patients as soon as DO was observed during cystometry failed to suppress detrusor contractions. ${ }^{40}$ However, another study showed a significant increase in both the volume of the first involuntary DO and the mean CMC in 29 patients with multiple sclerosis stimulated with PTNS. ${ }^{41}$ Studies examining pre- and posttreatment urodynamic data in non-neurogenic $\mathrm{OAB}$ also show contradictory results. Vandonick et al found complete suppression of DO in only a few cases, while others report elimination of DO in $76.9 \% .{ }^{42,43}$ Nevertheless, in both these studies, CMC increased significantly. Furthermore, patients without $\mathrm{DO}$ at baseline were 1.7 times more prone to respond to PTNS than those with urodynamic proven DO. ${ }^{42}$

Peters et al performed the pivotal study on PTNS. In a multicenter, double-blind randomized controlled trial (RCT) (SUmiT trial), 12 weeks of PTNS was compared to sham stimulation. This was the first study including a validated sham arm providing more information on the placebo effect. In total, 220 patients were included. Outcome parameters were improvement in global response assessment (GRA), frequency voiding charts (FVC) data, and QoL. The GRA is a self-reported 7-point scale measuring the individual perception of treatment changes. Success was defined as moderate or marked improvement in the GRA. Patients receiving PTNS showed 55\% moderate or marked improvement compared to $21 \%$ in the sham group. After 12 weeks, FVC parameters showed significant improvements in frequency, nocturia, voids with moderate/severe urgency, and UUI in the PTNS group compared to the sham group ${ }^{36}$ Finazzi-Agro et al performed another sham-controlled RCT with similar results. ${ }^{35}$

Interestingly, there is no standardized treatment regimen. Different protocols are described $(3,6,8,12$ weeks) with the most objective data to be in favor of the 12-week regimen based on previously mentioned RCTs. ${ }^{35,36}$ However, in the study of Peters et al weekly sessions were given compared to sessions 3 times a week in the study of Finazzi-Agro et al. Given the fact that both the studies show positive results, stimulation once a week seems to be effective and less timeconsuming. Shorter schedules are reported by Yoong et al who found an overall $67.5 \%$ positive response in 43 women who received a shortened 6-week PTNS treatment protocol with a 50\% symptom reduction and a $25 \%$ improvement in QoL, which is broadly comparable to the conventional 12-week results. ${ }^{44}$ However, with the 6-week regimen, the median time to relapse was 3 weeks so the authors concluded that it was more cost-effective to provide 12 weekly sessions in newly diagnosed patients.

While most objective data published favor a 12-week protocol, shortened regimes might also be effective, but perhaps less sustainable. Until further data are published, treatment protocols remain mainly dependent on individual patient-physician preferences.

Relapse of symptoms after successful treatment is likely to occur after PTNS. In a study on 11 patients with a successful outcome of 12-week PTNS, an interval of 6 weeks without stimulation was introduced. After this interval, 7 of 11 (63\%) experienced a $\geq 50 \%$ worsening of their complaints, which returned to baseline after re-starting PTNS. ${ }^{45}$

Yoong et $\mathrm{a} \mathrm{l}^{44}$ published the 2-year follow-up of 23 patients without deterioration of initially achieved results if a maintenance schedule of PTNS was given. Their study consisted of an open-door policy whereby patients could receive PTNS whenever they felt it necessary. A median of 8.42 treatments per year with a median length between the treatments of 64.3 days was given. Nocturnal frequency decreased with 57\%. Daytime frequency and UUI episodes at 2 years were significantly lower than at pretreatment ( $6.6 \mathrm{vs} 11.8$ and $2.0 \mathrm{vs} 3.5$, respectively; $p<0.05$ ) and comparable to those at 6 weeks. The median satisfaction score after 2 years of maintenance PTNS therapy was comparable to that recorded at 6 weeks $(7.25 / 10$ vs $9.6 / 10 ; p=0.25) .{ }^{44}$

Peters et $\mathrm{al}^{46}$ followed 50 participants from the SUmiT Trial who met the primary effectiveness endpoint after 
12 weekly PTNS. These patients were prescribed a fixedschedule 14-week tapering protocol followed by a personal treatment. Of this group, 29 patients completed the 36-month protocol receiving a median of 1.1 treatments per month. At 3 years, $77 \%$ remained relapse free with 8.7 median voids per day (baseline 12.0) and 0.3 UUI episodes per day (baseline 3.3). QoL remained markedly improved from baseline through 3 years. ${ }^{46}$ Other studies confirm sustainability with a maintenance schedule. ${ }^{47,48}$ Maintaining PTNS once every 2 or 3 weeks seems to be sufficient to sustain therapeutic effect in those patients who benefit from PTNS.

\section{Side effects}

From the studies examined, it was found that PTNS has no serious adverse events. Side effects described in the literature are mild and mainly related to needle insertion, bruising $(0.9 \%)$, discomfort (1.8\%), and slight bleeding. ${ }^{34-36}$

\section{PTNS compared to other treatments}

Several studies aimed to establish the effectiveness of PTNS compared to other treatments (Table 1). Two RCTs compared PTNS versus tolterodine in patients with non-neurogenic OAB. ${ }^{49,50}$ UUI decreased significantly after 3 months in both the groups and QoL increased. However, in both the RCTs, no significant difference was seen between both the treatments regarding QoL, 24-hour voiding episodes and UUI. Fewer side effects were seen in the PTNS group. ${ }^{49,50}$ Other antimuscarinics compared to PTNS yielded similar outcomes. ${ }^{14,51}$ A large Cochrane review compared seven trials with various types of peripheral neuromodulation (intravaginal ES, PTNS/SANS, and transcutaneous nerve stimulation) to antimuscarinics. Subjective improvement rates were observed in favor of ES. In 54\%, no improvement was seen with antimuscarinics versus $33 \%$ with ES (risk ratio $0.64,95 \%$ CI 1.15-2.34). However, this was significant only for PTNS (risk ratio 2.21, 95\% CI 1.13-4.33) and was not supported by significant changes in voiding parameter or QoL. The authors concluded that antimuscarinics were a well-established therapy for OAB, and limited evidence from small trials might suggest ES to be a better option in patients refractory to antimuscarinics. ${ }^{52}$

A subsequent Cochrane review compared ES with nonimplantable electrodes for OAB to no treatment or other available treatments. They included 63 studies and found moderate-quality evidence indicating that ES was better for the perception of improvement of OAB symptoms than pelvic muscle floor training (PMFT) (risk ratio [RR] 1.60, 95\% CI 1.19-2.14), drug treatment (RR 1.20,95\% CI 1.04-1.38) and placebo or sham treatment (RR 2.26, 95\% CI 1.85-2.77). The authors concluded that ES has better results than PMFT. Low evidence suggested participants receiving ES plus PMFT, compared to PMFT training alone were more likely to report improvement in UUI (RR 2.82, 95\% CI 1.44-5.52). They concluded that ES shows promising results compared to no treatment, placebo/sham treatment, PMFT, and drug treatment and that adding ES to other treatments might be beneficial. ${ }^{53}$

Sancaktar et $\mathrm{al}^{54}$ also studied PTNS as part of a multimodal treatment. They compared antimuscarinics with or without PTNS in 40 women with OAB. Frequency decreased from $12.8 \pm 1.3$ at baseline to $6.4 \pm 0.6$ in the antimuscarinics group and $12.2 \pm 1.2$ to $4.5 \pm 0$ in the antimuscarinics/PTNS group $(p<0.05)$. Urgency, UUI, and QoL improved in both the groups but was significantly better if multimodal treatment was given. ${ }^{54}$ In a study by Karademir et al, the combination of PTNS and oxybutinine led to an overall response of $83.2 \%$ compared to $61.6 \%$ response rate with PTNS alone; however, this difference was not significant $(p=0.24) .{ }^{55}$ Other studies were also unable to confirm the additional effects of antimuscarinics with PTNS. ${ }^{56}$

Patients with $\mathrm{OAB}$, especially when refractory to first-line treatments, pose a therapeutic challenge. Efficacy of antimuscarinics may be limited by their intolerable side effects and/or inadequate response. Furthermore, a high number of patients discontinue antimuscarinics on the long term. One study showed that the adherence rates for tolterodine and oxybutinine after 12 months were $9 \%$ and $6 \%$, respectively. ${ }^{57}$ PTNS might be a good alternative treatment. Other options include SNS or Botox. However, to our knowledge, no RCT has compared these second-line treatments.

\section{Prognostic factors for PTNS}

Few data are available on the prognostic factors for PTNS. Urodynamic studies in patients with OAB receiving PTNS suggest better treatment outcome in those patients without actual DO. ${ }^{42}$ In a study of 132 patients, numerous clinical parameters were evaluated and most of them could not reveal any prognostic value. ${ }^{58}$ Even a history of sexual and/ or physical abuse did not alter PTNS treatment outcome. The only factor that seemed to show any influence was a low total score at baseline in the SF-36 general QoL questionnaire. This proved to be predictive for not obtaining objective or subjective success. Patients with a low SF-36 Mental Component Summary were especially prone to fail. These patients also scored worse on disease-specific QoL questionnaires, despite that they had no difference 


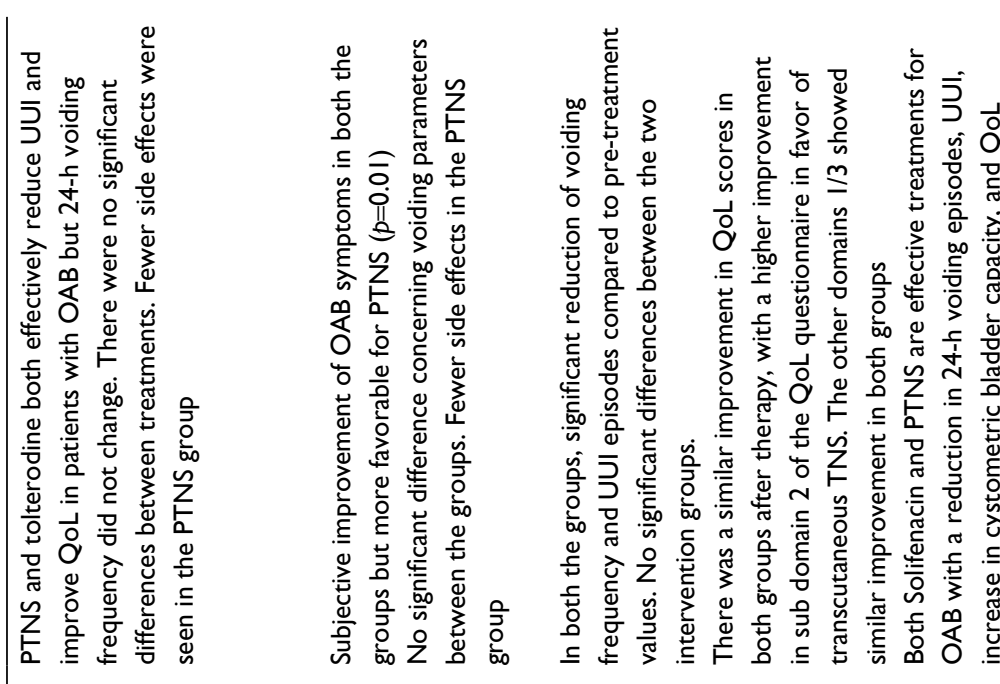

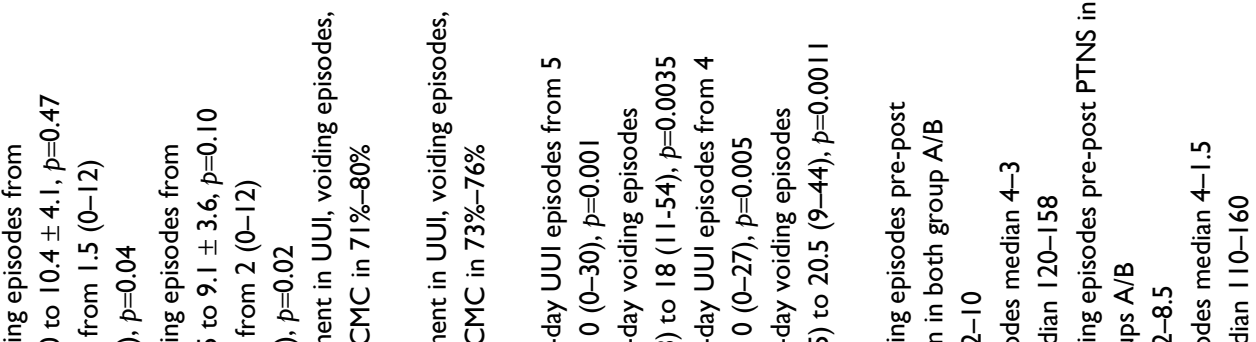

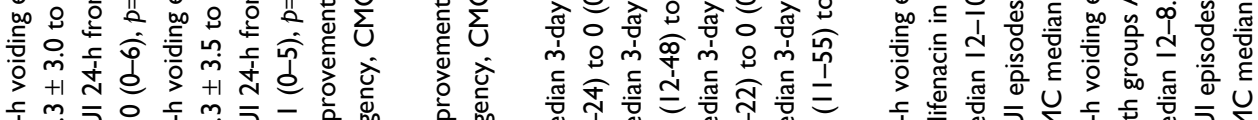

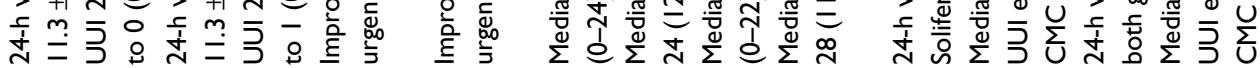

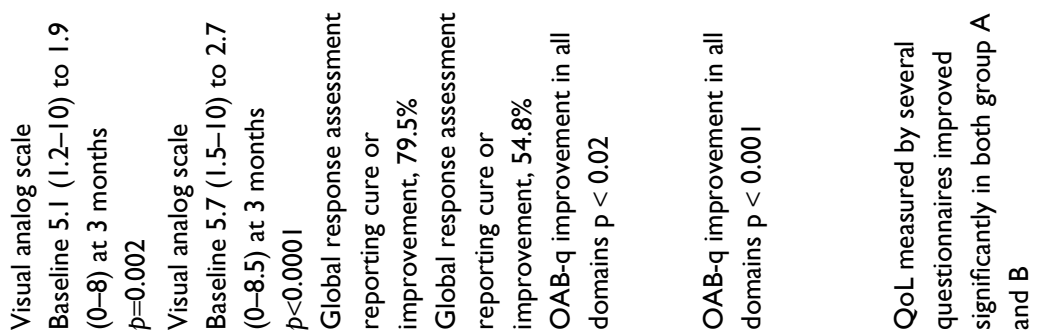

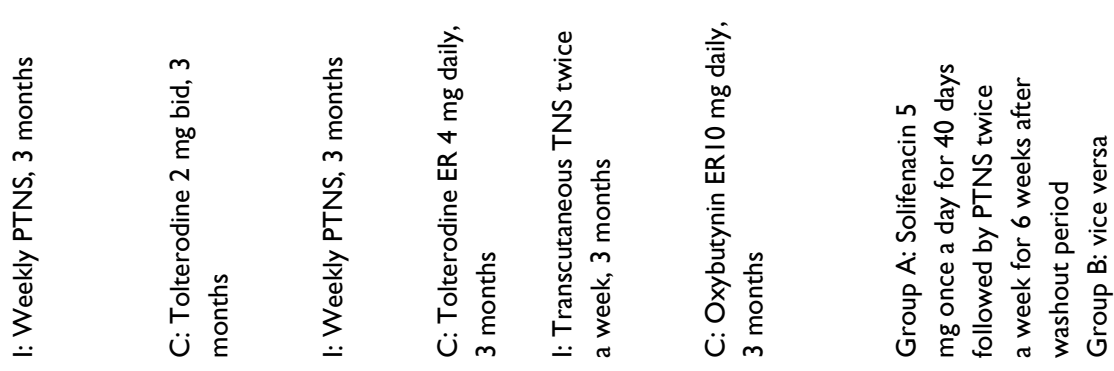

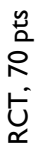

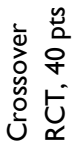

$\frac{0}{\pi}$
$\frac{0}{0}$
$\frac{n}{0}$
$\frac{0}{0}$
0

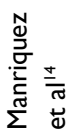

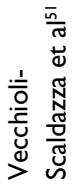




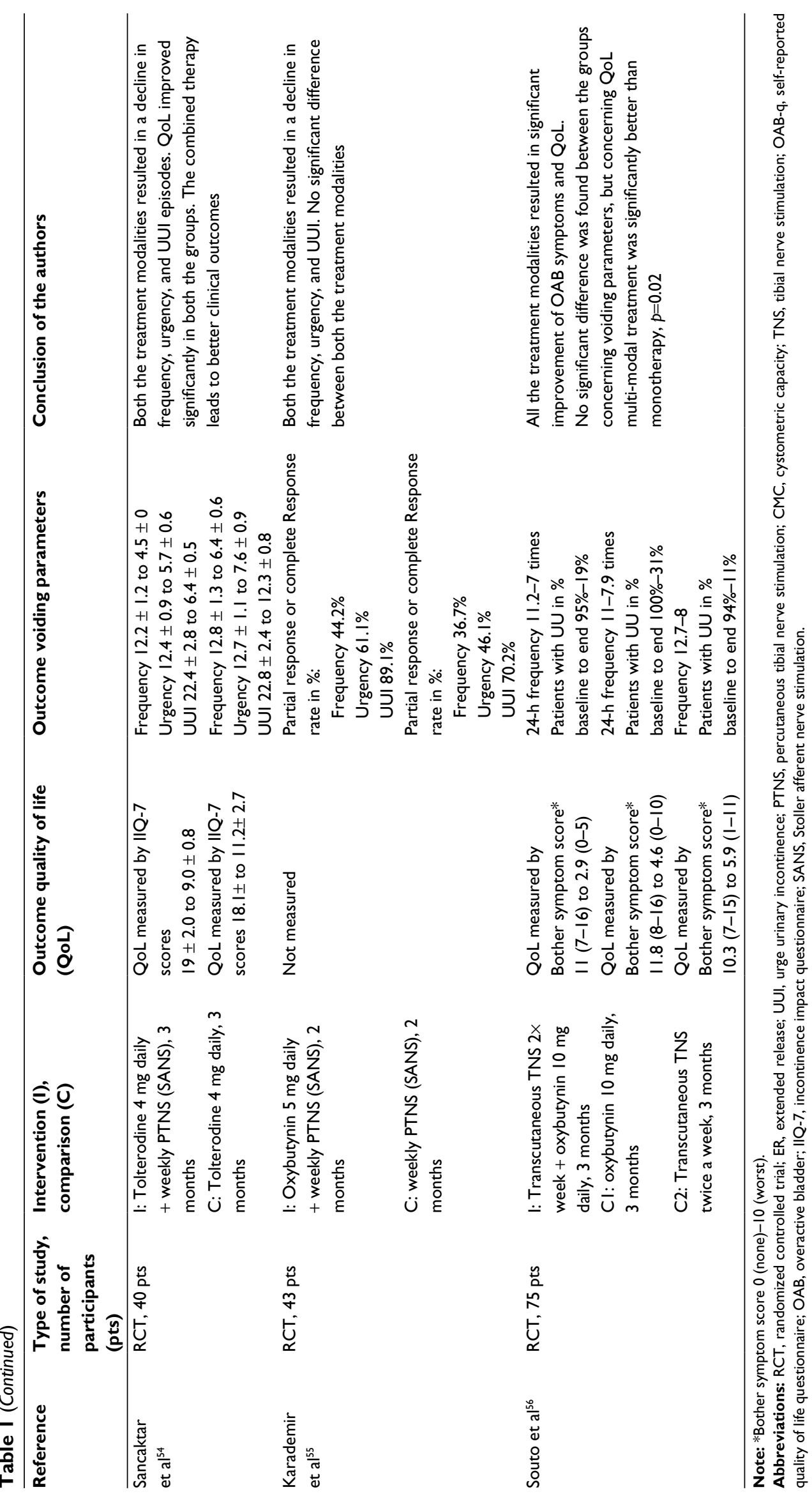


in disease severity compared to patients with good mental health. ${ }^{58}$

Therefore, mental health questionnaires might be helpful as an additional tool to select optimal candidates for PTNS.

\section{Patient perspectives}

Some studies evaluated actual patient preferences concerning treatment options for refractory OAB.$^{59,60} \mathrm{~A}$ best-worst scaling, together with surveys with different individual attributes, was used to assess the preferences of 245 patients from the USA and the UK. Most patients (98.8\%) were willing to try at least one of the different treatments. On a scale from $0 \%$ to $100 \%$, the mean percentage likelihoods of trying SNS, Botox, and PTNS were $45 \%, 43 \%$, and $62 \%$, respectively. The main attributes in general considered important in decision-making were "lasting improvement," "minimal side effects," and "send signals to the brain." Worst-rated attributes were "be willing to catheterize" and "complications of implant." However, preferences for the attributes differed mainly based on which treatment patients preferred; for example, patients preferring PTNS favored the attributes "needle insertion in the ankle" and "multiple visits required" more than patients favoring SNS. 59,60

Thus, incorporation of a decision tool addressing these attributes might help patients to increase compliance, treatment effect, and satisfaction.

\section{Cost-effectiveness}

Patient with refractory OAB remain a therapeutic challenge, and the effectiveness of second-line treatment options should be weighed against their costs. An economic model comparing SNS with Botox was developed using a probabilistic Markov analytic model in Dutch patients with refractory OAB. Different modeling scenarios were used. The 5-year costs were $€ 25,780$ for SNS and $€ 19,353$ for Botox, leading to $€ 6,428$ additional costs per patient for SNS. SNS became cost-effective (incremental cost-effectiveness ratio [ICER] $<40,000)$ from the third yearly treatment of Botox onward if given under general anesthesia. ${ }^{61} \mathrm{~A}$ comparable study conducted in Italy calculated the 10-year costs for SNS at $€ 32,975$ versus $€ 33,309$ for Botox, with cumulative quality-adjusted life years (QALYs) of 7.52 and 6.93, respectively. It was concluded that the relatively higher initial costs of early SNS can be offset by the favorable long-term outcomes. ${ }^{62}$ Staskin et $\mathrm{al}^{63}$ compared the costs of different treatment modalities for OAB in the USA. PTNS was the least costly ( $\$ 4,999$ for a 3-year treatment), followed by Botox $(\$ 7,651)$ and SNS $(\$ 26,269)$ for the same treatment period. ${ }^{63}$ Martinson et al also concluded that PTNS had substantially lower costs compared to SNS in the USA. Furthermore, an additional $1 \%$ of patients would remain on therapy at 2 years if SNS was used rather than PTNS, but average additional costs per patient would be $>\$ 500,000 .{ }^{64}$ Despite variation between countries PTNS appears not to be cost-effective as a primary treatment option compared to antimuscarinics but might be a good alternative in therapy refractory patients. However, on the long term, SNS might be more cost-effective considering the necessity of repeated clinical visits for PTNS.

\section{Implantable PTNS}

While maintenance therapy seems necessary to sustain therapeutic effect, soon after its clinical introduction, it was realized that repeated visits would finally lead to a logistic problem. Besides overfull clinics, the travel burden for patients would be high and PTNS treatment-on-demand would be impossible. Transcutaneous stimulation was tried with surface electrodes, but results indicate that it might be less effective because of the impendence of the skin. ${ }^{65} \mathrm{~A}$ new promising development came with the introduction of an implantable stimulator near the ankle. Van der Pal et $\mathrm{al}^{66}$ were the first to study the subcutaneous implant Urgent-SQ (Uroplasty, Inc, Minnetonka, MN, USA) in 8 patients with refractory OAB. The Urgent-SQ was surgically implanted $\sim 5 \mathrm{~cm}$ above the medial malleolus near the PTN, without actually exposing it. During the procedure, the implant was activated to confirm the correct position. Motor and sensory responses were evaluated postoperatively at day 10 and after $3-6-12$ months. The primary objective was $\geq 50 \%$ reduction in the number of UUI and/or voids on bladder diary. At 3 , 6 , and 12 months, 5, 6, and 4 patients, respectively, met the primary objective. At 3-and 6-month follow-up, voiding and QoL parameters had significantly improved in these patients; at 12 months, it remained stable compared to 6 months. UTI temporary walking difficulties and spontaneous radiating sensations were reported as adverse events, and there was no local infection, erosion, or dislocation. As in studies concerning sacral stimulation, not all patients who respond well to PTNS have similar results with implantable devices. For example, in one patient, the implant was removed after 12 months because of technical failure. During the procedure, the implant was activated which did not result in a motor response. The device was examined but results not mentioned. ${ }^{66}$

Janssen et $\mathrm{al}^{67}$ published the long-term efficacy and safety of these patients in an open-label study. The 7 patients with the implant still in situ were contacted after 9 years and evaluated with an interview, physical examination, ankle 
X-ray, FVC, questionnaires addressing adverse events, performance, efficacy, safety, and QoL. Results showed that 6 of the 7 patients still had sensory and locomotor responses on stimulation at 9-year follow-up. Also, 3 patients, who had a successful treatment response at 1 year, still used the device. The implants were intact without migration and/or displacement; 2 patients experienced minor discomfort. The conclusion was that, after 9 years, the Urgent-SQ implant was a safe device and well tolerated. ${ }^{67}$

Results of a new tibial implanted device (BlueWind Medical, Herzliya, Israel) have recently been published. ${ }^{68}$ The installation procedure resembled that of the UrgentSQ. For the 15 patients in whom the device was implanted, a significant improvement was seen in both frequency and UUI. At 3-months follow-up, a significant change was seen in 24-hour voiding frequency from a mean 11.8 (SD 3.5) to 8.1 (SD 2.0) per day ( $p=0.002$ ). The number of severe UUI episodes decreased from 2.8 (SD 5.2) to 0.3 (SD 0.4) episodes a day ( $p=0.017)$. After implantation, 3 patients had prolonged antibiotic treatment and 3 patients needed prolonged pain treatment for 1 week. In 1 patient, the device was explanted due to pain and swelling suspicious for infection, although tissue cultures did not reveal a bacterial infection. ${ }^{68}$

Implantable devices are well tolerated and safe without long-term complications. Although pilot studies show promising results, more research is necessary to establish further therapeutic value.

\section{PTNS in children}

$\mathrm{OAB}$ is also common in the pediatric population aged 6-16 years. If standard treatment options (eg, urotherapy, PMFT, and antimuscarinics) fail, PTNS might be a good option. De Genarro et $\mathrm{al}^{69}$ found that PTNS was well tolerated in 23 children (4-17 years) with refractory lower urinary tract symptoms (LUTS). In 71\%, symptoms improved and low scores on the visual analog scale for pain were noted, which decreased even further during the 12-week treatment period. ${ }^{69}$

Several sham-controlled studies have been conducted using (mainly) transcutaneous stimulation. In a double-blind RCT, 20 children with refractory $\mathrm{OAB}$ were given either transcutaneous TNS or sham treatment for 12 consecutive weeks. Pre- and post-urodynamic parameters were compared, and UUI were noted and scored with a range of 0-13 (from good to poor). Clinical results were defined as poor ( $\leq 3$-point decrease), medium (3-5-point decrease), good (6-8-point decrease), and very good (final score of $0-3$ ). In the PTNS group, very good results were seen in $45 \%$ compared to $66 \%$ in the sham group, and poor results in $45 \%$ versus $33 \%$, respectively.
Urodynamic parameters revealed significant improvement of volume voided during urgency $(184-265 \mathrm{~mL})$, maximum CMC (215-274 mL), and volume at onset of first DO (48-174 mL). The authors stated that, even though urodynamic data show improvement, subjective data remain the same and the placebo effect plays an important role. ${ }^{70}$ In a study by Hagstroem et al parasacral stimulation was compared with sham treatment. After 4 weeks of intervention, 61\% reported a decrease in incontinence severity versus only $2 \%$ in the sham group. However, no differences were seen in maximal and average voided volumes or urodynamic data. ${ }^{71,72}$ Another group studied the additional effect of parasacral stimulation in patients receiving urotherapy $;^{73} 62$ children with OAB were randomized either to urotherapy alone or combined with parasacral stimulation. In the standard group, $46 \%$ were completely dry versus $67 \%$ in the combined group; this was not significant. Furthermore, no differences were seen between both the groups concerning FVC parameters. The authors concluded that parasacral stimulation had no additional effect. ${ }^{73}$

Long-term outcome seems to be good in children treated with PTNS. In 44 children with LUTS, the cure rate after 1 year was $41 \%$ if being treated for $\mathrm{OAB}$ and $71 \%$ if being treated for dysfunctional voiding both defined according to the International Children's Continence Society, ${ }^{1}$ which remained stable after 2 years. Maintenance treatment was necessary in $29 \%$ of children with dysfunctional voiding and in $50 \%$ of children with $\mathrm{OAB} .^{74}$

Only one study compared PTNS versus parasacral stimulation and found a higher complete resolution of symptoms in the parasacral group versus the PTNS group (71\% vs 9\%) without significant differences in scores of urgency and UUI. However, because this was not an RCT, the data have to be interpreted with caution. ${ }^{13}$

Transcutaneous/percutaneous stimulation seems feasible in children with refractory LUTS. However, more trials with larger groups are necessary to determine the actual subjective and objective effect, while current data show conflicting results.

\section{Potential limitations}

Because this review has a non-systematic design, there are some limitations. Although a comprehensive search was made to include eligible articles, some potential articles could have been missed. Especially relevant non-English language studies might not have been included. Also, no methodological assessment or data extraction was done to detect heterogeneity or publication bias. Therefore, our conclusion needs to be interpreted with caution. 


\section{Conclusion}

PTNS can indeed modulate the voiding and storage function of the bladder leading to an overall subjective improvement of symptoms in about $60 \%$ of the patients and $47 \%-56 \%$ improvement of FVC parameters with sustainable outcome on the long run. The placebo effect (subjective improvement measured by patients who actually received sham treatment) is about $21 \%$ and may be even higher in children. This might be explained by the regular visits, peer-grouping, and/or the weekly attention paid to their problem by the caregivers. PTNS seems not to be cost-effective as a primary treatment compared to antimuscarinics, but is a good treatment option in refractory $\mathrm{OAB}$ or when antimuscarinics are not tolerated. Although PTNS is minimally invasive and not costly, it is time consuming. Therefore, new techniques with implants are being explored and show initially promising results.

\section{Disclosure}

Dr Heesakkers received grants and personal fees from Bluewind. The authors report no other conflicts of interest in this work.

\section{References}

1. Austin PF, Bauer SB, Bower W, et al. The standardization of terminology of lower urinary tract function in children and adolescents: update report from the standardization committee of the International Children's Continence Society. Neurourol Urodyn. 2016;35(4):471-481.

2. Haylen BT, de Ridder D, Freeman RM, et al. An International Urogynecological Association (IUGA)/International Continence Society (ICS) joint report on the terminology for female pelvic floor dysfunction. Neurourol Urodyn. 2010;29(1):4-20.

3. Milsom I, Coyne KS, Nicholson S, Kvasz M, Chen CI, Wein AJ. Global prevalence and economic burden of urgency urinary incontinence: a systematic review. Eur Urol. 2014;65(1):79-95.

4. Coyne KS, Wein A, Nicholson S, Kvasz M, Chen CI, Milsom I. Comorbidities and personal burden of urgency urinary incontinence: a systematic review. Int J Clin Pract. 2013;67(10):1015-1033.

5. Coyne KS, Kvasz M, Ireland AM, Milsom I, Kopp ZS, Chapple CR. Urinary incontinence and its relationship to mental health and healthrelated quality of life in men and women in Sweden, the United Kingdom, and the United States. Eur Urol. 2012;61(1):88-95.

6. Bartoli S, Aguzzi G, Tarricone R. Impact on quality of life of urinary incontinence and overactive bladder: a systematic literature review. Urology. 2010;75(3):491-500.

7. Zorn BH, Montgomery H, Pieper K, Gray M, Steers WD. Urinary incontinence and depression. J Urol. 1999;162(1):82-84.

8. Bower WF. Self-reported effect of childhood incontinence on quality of life. J Wound Ostomy Continence Nurs. 2008;35(6):617-621.

9. Natale N, Kuhn S, Siemer S, Stockle M, von Gontard A. Quality of life and self-esteem for children with urinary urge incontinence and voiding postponement. J Urol. 2009;182(2):692-698.

10. Sampaio AS, Fraga LG, Salomao BA, et al. Are lower urinary tract symptoms in children associated with urinary symptoms in their mothers? J Pediatr Urol. 2017;13(3):e1-269.e6.

11. de Groat WC, Yoshimura N. Anatomy and physiology of the lower urinary tract. Handb Clin Neurol. 2015;130:61-108.
12. de Groat WC, Griffiths D, Yoshimura N. Neural control of the lower urinary tract. Compr Physiol. 2015;5(1):327-396.

13. Barroso U Jr, Viterbo W, Bittencourt J, Farias T, Lordelo P. Posterior tibial nerve stimulation vs parasacral transcutaneous neuromodulation for overactive bladder in children. J Urol. 2013;190(2):673-677.

14. Manriquez V, Guzman R, Naser M, et al. Transcutaneous posterior tibial nerve stimulation versus extended release oxybutynin in overactive bladder patients. A prospective randomized trial. Eur J Obstet Gynecol Reprod Biol. 2016;196:6-10.

15. Schreiner L, dos Santos TG, Knorst MR, da Silva Filho IG. Randomized trial of transcutaneous tibial nerve stimulation to treat urge urinary incontinence in older women. Int Urogynecol J. 2010;21(9): $1065-1070$

16. Bower WF, Moore KH, Adams RD, Shepherd R. A urodynamic study of surface neuromodulation versus sham in detrusor instability and sensory urgency. J Urol. 1998;160(6 Pt 1):2133-2136.

17. Fowler CJ, Griffiths D, de Groat WC. The neural control of micturition. Nat Rev Neurosci. 2008;9(6):453-466.

18. Ferroni MC, Slater RC, Shen B, et al. Role of the brain stem in tibial inhibition of the micturition reflex in cats. Am J Physiol Renal Physiol. 2015;309(3):F242-F250.

19. Fall M, Lindstrom S. Electrical stimulation. A physiologic approach to the treatment of urinary incontinence. Urol Clin North Am. 1991;18(2):393-407.

20. Liao KK, Chen JT, Lai KL, et al. Effect of sacral neuromodulation on the spinal nociceptive reflex of patients with idiopathic overactive bladder. Neuromodulation. 2008;11(1):50-55.

21. Bandari J, Bansal U, Zhang Z, et al. Neurotransmitter mechanisms underlying sacral neuromodulation of bladder overactivity in cats. Neuromodulation. 2017;20(1):81-87.

22. Lyon TD, Ferroni MC, Kadow BT, et al. Pudendal but not tibial nerve stimulation inhibits bladder contractions induced by stimulation of pontine micturition center in cats. Am J Physiol Regul Integr Comp Physiol. 2016;310(4):R366-R374.

23. Tai C, Larson JA, Ogagan PD, et al. Differential role of opioid receptors in tibial nerve inhibition of nociceptive and nonnociceptive bladder reflexes in cats. Am J Physiol Renal Physiol. 2012;302(9):F1090-F1097.

24. Li X, Liao L, Chen G, Wang Z, Deng H. Involvement of opioid receptors in inhibition of bladder overactivity induced by sacral neuromodulation in pigs: a possible action mechanism. Neurourol Urodyn. 2016:1-7.

25. Blok BF, Groen J, Bosch JL, Veltman DJ, Lammertsma AA. Different brain effects during chronic and acute sacral neuromodulation in urge incontinent patients with implanted neurostimulators. BJU Int. 2006;98(6):1238-1243.

26. Braun PM, Baezner H, Seif C, et al. Alterations of cortical electrical activity in patients with sacral neuromodulator. Eur Urol. 2002;41(5):562-566; discussion 566-567.

27. Finazzi-Agro E, Rocchi C, Pachatz C, et al. Percutaneous tibial nerve stimulation produces effects on brain activity: study on the modifications of the long latency somatosensory evoked potentials. Neurourol Urodyn. 2009;28(4):320-324.

28. Stux G. General standards in acupuncture treatment of chronic pain. Schmerz. 1997;11(2):126-127.

29. Minni B, Capozza N, Creti G, De Gennaro M, Caione P, Bischko J. Bladder instability and enuresis treated by acupuncture and electrotherapeutics: early urodynamic observations. Acupunct Electrother Res. 1990;15(1):19-25.

30. van Voskuilen AC, Oerlemans DJ, Weil EH, de Bie RA, van Kerrebroeck PE. Long term results of neuromodulation by sacral nerve stimulation for lower urinary tract symptoms: a retrospective single center study. Eur Urol. 2006;49(2):366-372.

31. van Kerrebroeck PE, van Voskuilen AC, Heesakkers JP, et al. Results of sacral neuromodulation therapy for urinary voiding dysfunction: outcomes of a prospective, worldwide clinical study. J Urol. 2007;178(5): 2029-2034.

32. McGuire EJ, Zhang SC, Horwinski ER, Lytton B. Treatment of motor and sensory detrusor instability by electrical stimulation. $J$ Urol. 1983;129(1):78-79. 
33. Stoller ML, Copeland S, Millard RJ, Murnaghan GF. The efficacy of acupuncture in reversing the unstable bladder in pig-tailed monkeys. J Urol. 1987;137(4):A104-A104.

34. Govier FE, Litwiller S, Nitti V, Kreder KJ Jr, Rosenblatt P. Percutaneous afferent neuromodulation for the refractory overactive bladder: results of a multicenter study. J Urol. 2001;165(4):1193-1198.

35. Finazzi-Agro E, Petta F, Sciobica F, Pasqualetti P, Musco S, Bove P. Percutaneous tibial nerve stimulation effects on detrusor overactivity incontinence are not due to a placebo effect: a randomized, double-blind, placebo controlled trial. J Urol. 2010;184(5):2001-2006.

36. Peters KM, Carrico DJ, Perez-Marrero RA, et al. Randomized trial of percutaneous tibial nerve stimulation versus Sham efficacy in the treatment of overactive bladder syndrome: results from the SUmiT trial. J Urol. 2010;183(4):1438-1443.

37. van Balken MR, Vandoninck V, Gisolf KW, et al. Posterior tibial nerve stimulation as neuromodulative treatment of lower urinary tract dysfunction. J Urol. 2001;166(3):914-918.

38. Vandoninck V, Van Balken MR, Finazzi Agro E, et al. Posterior tibial nerve stimulation in the treatment of urge incontinence. Neurourol Urodyn. 2003;22(1):17-23.

39. van der Pal F, van Balken MR, Heesakkers JP, Debruyne FM, Kiemeney LA, Bemelmans BL. Correlation between quality of life and voiding variables in patients treated with percutaneous tibial nerve stimulation. BJU Int. 2006;97(1):113-116.

40. Fjorback MV, van Rey FS, van der Pal F, Rijkhoff NJ, Petersen T, Heesakkers JP. Acute urodynamic effects of posterior tibial nerve stimulation on neurogenic detrusor overactivity in patients with MS. Eur Urol. 2007;51(2):464-470; discussion 471-462.

41. Kabay SC, Yucel M, Kabay S. Acute effect of posterior tibial nerve stimulation on neurogenic detrusor overactivity in patients with multiple sclerosis: urodynamic study. Urology. 2008;71(4):641-645.

42. Vandoninck V, van Balken MR, Finazzi Agro E, et al. Percutaneous tibial nerve stimulation in the treatment of overactive bladder: urodynamic data. Neurourol Urodyn. 2003;22(3):227-232.

43. Klingler HC, Pycha A, Schmidbauer J, Marberger M. Use of peripheral neuromodulation of the $\mathrm{S} 3$ region for treatment of detrusor overactivity: a urodynamic-based study. Urology. 2000;56(5):766-771.

44. Yoong W, Shah P, Dadswell R, Green L. Sustained effectiveness of percutaneous tibial nerve stimulation for overactive bladder syndrome: 2-year follow-up of positive responders. Int Urogynecol J. 2013;24(5): 795-799.

45. van der Pal F, van Balken MR, Heesakkers JP, Debruyne FM, Bemelmans BL. Percutaneous tibial nerve stimulation in the treatment of refractory overactive bladder syndrome: is maintenance treatment necessary? BJU Int. 2006;97(3):547-550.

46. Peters KM, Carrico DJ, Wooldridge LS, Miller CJ, MacDiarmid SA. Percutaneous tibial nerve stimulation for the long-term treatment of overactive bladder: 3-year results of the STEP study. J Urol. 2013;189(6):2194-2201.

47. Canbaz Kabay S, Kabay S, Mestan E, et al. Long term sustained therapeutic effects of percutaneous posterior tibial nerve stimulation treatment of neurogenic overactive bladder in multiple sclerosis patients: 12-months results. Neurourol Urodyn. 2017;36(1):104-110.

48. MacDiarmid SA, Peters KM, Shobeiri SA, et al. Long-term durability of percutaneous tibial nerve stimulation for the treatment of overactive bladder. J Urol. 2010;183(1):234-240.

49. Preyer O, Umek W, Laml T, et al. Percutaneous tibial nerve stimulation versus tolterodine for overactive bladder in women: a randomised controlled trial. Eur J Obstet Gynecol Reprod Biol. 2015;191:51-56.

50. Peters KM, Macdiarmid SA, Wooldridge LS, et al. Randomized trial of percutaneous tibial nerve stimulation versus extended-release tolterodine: results from the overactive bladder innovative therapy trial. $J$ Urol. 2009;182(3):1055-1061.

51. Vecchioli-Scaldazza C, Morosetti C, Berouz A, Giannubilo W, Ferrara $\mathrm{V}$. Solifenacin succinate versus percutaneous tibial nerve stimulation in women with overactive bladder syndrome: results of a randomized controlled crossover study. Gynecol Obstet Invest. 2013;75(4): 230-234.
52. Rai BP, Cody JD, Alhasso A, Stewart L. Anticholinergic drugs versus non-drug active therapies for non-neurogenic overactive bladder syndrome in adults. Cochrane Database Syst Rev. 2012;12:CD003193.

53. Stewart F, Gameiro LF, El Dib R, Gameiro MO, Kapoor A, Amaro JL. Electrical stimulation with non-implanted electrodes for overactive bladder in adults. Cochrane Database Syst Rev. 2016;12:CD010098.

54. Sancaktar M, Ceyhan ST, Akyol I, et al. The outcome of adding peripheral neuromodulation (Stoller afferent neuro-stimulation) to antimuscarinic therapy in women with severe overactive bladder. Gynecol Endocrinol. 2010;26(10):729-732.

55. Karademir K, Baykal K, Sen B, Senkul T, Iseri C, Erden D. A peripheric neuromodulation technique for curing detrusor overactivity: Stoller afferent neurostimulation. Scand J Urol Nephrol. 2005;39(3):230-233.

56. Souto SC, Reis LO, Palma T, Palma P, Denardi F. Prospective and randomized comparison of electrical stimulation of the posterior tibial nerve versus oxybutynin versus their combination for treatment of women with overactive bladder syndrome. World J Urol. 2014;32(1): 179-184.

57. Shaya FT, Blume S, Gu A, Zyczynski T, Jumadilova Z. Persistence with overactive bladder pharmacotherapy in a Medicaid population. Am $J$ Manag Care. 2005;11(4 Suppl):S121-S129.

58. van Balken MR, Vergunst H, Bemelmans BL. Prognostic factors for successful percutaneous tibial nerve stimulation. Eur Urol. 2006;49(2):360-365.

59. Beusterien K, Kennelly MJ, Bridges JF, Amos K, Williams MJ, Vasavada S. Use of best-worst scaling to assess patient perceptions of treatments for refractory overactive bladder. Neurourol Urodyn. 2016;35(8): 1028-1033.

60. Hashim H, Beusterien K, Bridges JF, Amos K, Cardozo L. Patient preferences for treating refractory overactive bladder in the UK. Int Urol Nephrol. 2015;47(10):1619-1627.

61. Leong RK, de Wachter SG, Joore MA, van Kerrebroeck PE. Costeffectiveness analysis of sacral neuromodulation and botulinum toxin A treatment for patients with idiopathic overactive bladder. BJU Int. 2011;108(4):558-564.

62. Bertapelle MP, Vottero M, Popolo GD, et al. Sacral neuromodulation and botulinum toxin A for refractory idiopathic overactive bladder: a cost-utility analysis in the perspective of Italian Healthcare System. World J Urol. 2015;33(8):1109-1117.

63. Staskin DR, Peters KM, MacDiarmid S, Shore N, de Groat WC. Percutaneous tibial nerve stimulation: a clinically and cost effective addition to the overactive bladder algorithm of care. Curr Urol Rep. 2012;13(5):327-334.

64. Martinson M, MacDiarmid S, Black E. Cost of neuromodulation therapies for overactive bladder: percutaneous tibial nerve stimulation versus sacral nerve stimulation. $J$ Urol. 2013;189(1):210-216.

65. Andrews BJ, Reynard JM. Transcutaneous posterior tibial nerve stimulation for treatment of detrusor hyperreflexia in spinal cord injury. $J$ Urol. 2003;170(3):926

66. van der Pal F, van Balken MR, Heesakkers JP, Debruyne FM, Bemelmans BL. Implant-driven tibial nerve stimulation in the treatment of refractory overactive bladder syndrome: 12-month follow-up. Neuromodulation. 2006;9(2):163-171.

67. Janssen DA, Farag F, Heesakkers JP. Urgent-SQ implant in treatment of overactive bladder syndrome: 9-year follow-up study. Neurourol Urodyn. 2013;32(5):472-475.

68. van Breda HM, Martens FM, Tromp J, Heesakkers JP. A new implanted posterior tibial nerve stimulator for the treatment of overactive bladder syndrome: 3-month results of a novel therapy at a single center. $J$ Urol. 2017;198(1):205-210.

69. De Gennaro M, Capitanucci ML, Mastracci P, Silveri M, Gatti C, Mosiello G. Percutaneous tibial nerve neuromodulation is well tolerated in children and effective for treating refractory vesical dysfunction. J Urol. 2004;171(5):1911-1913.

70. Boudaoud N, Binet A, Line A, et al. Management of refractory overactive bladder in children by transcutaneous posterior tibial nerve stimulation: a controlled study. J Pediatr Urol. 2015;11(3):138 e131-138 e110. 
71. Hagstroem S, Mahler B, Madsen B, Djurhuus JC, Rittig S. Transcutaneous electrical nerve stimulation for refractory daytime urinary urge incontinence. J Urol. 2009;182(4 Suppl):2072-2078.

72. Borch L, Rittig S, Kamperis K, Mahler B, Djurhuus JC, Hagstroem S. No immediate effect on urodynamic parameters during transcutaneous electrical nerve stimulation (TENS) in children with overactive bladder and daytime incontinence - a randomized, double-blind, placebocontrolled study. Neurourol Urodyn. 2016:1-7.
73. Sillen U, Arwidsson C, Doroszkiewicz M, et al. Effects of transcutaneous neuromodulation (TENS) on overactive bladder symptoms in children: a randomized controlled trial. J Pediatr Urol. 2014;10(6): $1100-1105$.

74. Capitanucci ML, Camanni D, Demelas F, Mosiello G, Zaccara A, De Gennaro M. Long-term efficacy of percutaneous tibial nerve stimulation for different types of lower urinary tract dysfunction in children. J Urol. 2009;182(4 Suppl):2056-2061.

\section{Publish your work in this journal}

Research and Reports in Urology is an international, peer-reviewed, open access journal publishing original research, reports, editorials, reviews and commentaries on all aspects of adult and pediatric urology in the clinic and laboratory including the following topics: Pathology, pathophysiology of urological disease; Investigation and treatment of

\section{Dovepress}

urological disease; Pharmacology of drugs used for the treatment of urological disease. The manuscript management system is completely online and includes a very quick and fair peer-review system, which is all easy to use. Visit http://www.dovepress.com/testimonials.php to read real quotes from published authors.

Submit your manuscript here: https://www.dovepress.com/research-and-reports-in-urology-journal 